\title{
Neuigkeiten vom Buch- und Zeitschriftenmarkt
}

\section{;6:51-55 Für die Schriftleitung: Eibe-Rudolf Rey}

Buchbesprechungen

Ziesing, Frank; Pfingsten, Ulrich

Selbstveränderung - Verhaltenstherapie selbst erfahren

Tubingen, DGVT-Verlag, 1994159 S., DM 36-ISBN 3-87159-403-2

Mit ihrem Ausbildungsmanual machen es sich die Autoren zum Ziel, ein Selbsterfahrungsprogramm für Patienten, Stu-denten und angehende

Verhaltenstherapeuten zu erstellen. Es soil dem Leser einen anschaulichen Eindruck des verhaltens-therapeutischen Ansatzes vermitteln und ihm ein Selbstverän-derungsprojekt - allein oder in einer Gruppe Gleichgesinnter - innerhalb von 12-16 Wochen ermöglichen. Im ersten Ab-schnitt leiten die Autoren den Leser zunächst unter anderem auch praktisch an, die dafür notwendigen Arbeitsbedingungen herzustellen (Kap. 1 und 2) und das gesetzte Ziel genau zu definieren (Kap. 3-5). Die weiteren Kapitel führen den Leser über den Weg einer ausführlichen Verhaltensanalyse mit dem Schwerpunkt der aufrechterhaltenden Bedingungen zum Er-klärungsmodell seiner Problematik (Kap. 6-8). Die weiteren Schritte bestehen in der Vermittlung von verhaltenstherapeu-tischen Methoden (Kap. 9-13), ihrer Einsatzmöglichkeiten und vor allem ihrer praktischen Umsetzung (Kap. 14-16). Problematisch erscheint dabei zuerst die Weite der Zielgruppe: Die Gruppe der angehenden Verhaltenstherapeuten scheint mir mit dem dargebotenen Stoff eher unterfordert, die Patienten-population dagegen überfordert. Ein Selbsterfahrungsprojekt, wie es von den Autoren konzipiert wird, erfordert fundierte Kenntnisse der Lerntheorie und speziell der Verhaltenstherapie, die durch Sekundärliteratur erworben werden müssen. Im erforderlichen Ausmaß ist dies für Patienten jedoch nicht zumutbar und - aufgrund mangelnder Kontrolle von Mißver-ständnissen - auch nicht sinnvoll. In Kombination mit vertie-fender Literatur liegt jedoch zumindest für Studenten ein brauchbares Manual vor. Die Idee, sich grundsätzlich im Rah-men des Selbsterfahrungsprojekts in die Rolle des Patienten zu begeben und in einer umfassenden Einzelfallstudie verhal-tenstherapeutische Behandlungsverfahren praktisch zu prü-fen, klingt interessant. Ein entsprechender «Selbstversuch» scheint lohnenswert. Zumal, wenn man die doch sehr theore-tisch ausgerichtete universitäre Ausbildung der Psychologen -und damit der zukünftigen Psychotherapeuten - bedenkt. Unter diesem Blickwinkel definieren die Autoren mit Mahoney «...Therapie als ein Wissen (...), in dem Menschen zu Wissen-schaftlern für die eigene Person ausgebildet werden». Entspre-chend bauen sie ihr Buch dann auch als eine Sammlung von Wissenstechniken auf. Leider laufen sie dabei oftmals Gefahr, ein Sammelsurium von - zweifellos sinnvollen - Techniken darzustellen, die für den noch ungeübten Leser ohne inneren Zusammenhang nebeneinander stehenbleiben. Der von den

Autoren angestrebte «spontane und kreative Umgang mit den Methoden» ist dabei ein zu hoch gestecktes Ziel, mit dem die Leserschaft überfordert wird. Man sollte vielleicht häufiger den «roten Faden mitliefern», das würde den Lesern das Pro-jekt erleichtern, ohne dem Anspruch an Selbständigkeit und Selbstverantwortlichkeit Abbruch zu tun. So ist durch die Leitfragen des Manuals, beispielsweise «Was will ich ändern?», eine gute und sinnvolle Gliederung vorgegeben. Leider werden diese Abschnitte zu wenig in den Fließtext eingebunden. Die Darstellung selbst ist dann jedoch in vielen Punkten sehr gut und eingängig formuliert. Besonders gelungen scheint mir in den Eingangskapiteln die Betonung und Begründung der für das Gelingen erforderlichen Projektbedingungen. Positiv ist unter anderem, daß sich die Autoren selbst an die genannten Maximen einer klaren Aufgabenstellung und konkreten Ausarbeitung halten! Erwähnenswert sind auch die vielen praktischen Hilfen zur Umsetzung der theoretischen Erkenntnisse ins Selbsterfahrungsprojekt. Dies ist besonders eindrucksvoll geschehen bei der Darstellung der Problemdefinition in Kapitel 3: Hier wird - fast in Form eines Entscheidungsbaumes -dem Leser vorgegeben, wie er die für ihn subjektiv erforderli-che Hilfestellung in gestaffelter Form aufbauen kann. Etwas befremdlich muten dann jedoch die beiden folgenden Kapitel an, die sehr ausführlich theoretisch aufbereitet wurden. Der Bezug zur Praxis fehlt hier! Es erhebt sich die Frage, warum es den Autoren ganz entgegen dem sonstigen Vor-gehen - erforderlich schien, den Leser mit theoretischen Begriffen geradezu zu bombardieren, anstatt die Technik der Verhaltensanalyse, die nachfolgend im Zentrum der Betrach-tung steht, in einfachen Worten einzuführen. Dies ware abso-lut ausreichend für die gegebene Zielsetzung. Die Kapitel 6 und 7 wirken danach etwas «eingeschoben» und ohne rechten Zusammenhang zu der in Kapitel 5 dargestellten Problemauswahl und Grundratenerhebung. Die Zusammenfüh-rung erfolgt dann in Kapitel 8, wo der Leser nun endlich erfährt, was er in den vorhergehenden Schritten erreicht haben sollte. Diese Gliederung scheint umständlich und vergrößert die Mühen des Lesers in unnötiger Weise. Eine kurze, klare Erklärung der weiteren Schritte im Sinne eines Ausblicks auf die Kapitel 4-8 ware hier weitaus sinnvofler. Dem Leser ware eine klare Ziel-definition und damit der genannte «rote Faden» gegeben, um die folgenden Kapitel sinnvoll in sein Projekt einzubinden. Eine Alternative ware auch - wie in späteren Kapiteln prakti-ziert - ein durchgängiges Beispiel zur Erstellung der Verhaltensanalyse. Dies würde ebenfalls den mangelnden Bezug zwischen den Kapiteln 4, 5 und 6 herstellen und der - anson-sten ausgezeichneten - Darstellung des SORCK-Schemas ihren zentralen Platz zuordnen. Der Rahmen dafür ware zweifellos vorhanden, ließe man nur die zum Teil überflüs-sigen theoretischen Eskapaden fallen und konzentrierte sich mehr auf praktische Relevanz.

Auch die alleinige Darstellung von klassischem und operan-tem Konditionieren als Lernparadigmen ist in diesem Sinne KARG ER @ 1996SKarger GmbH, Freiburg

-IIVJ Fax (0761) 4520714

etwas dürftig, wohingegen die historischen Aspekte eher zu viel Raum einnehmen. Dem Ziel der Praxisorientierung wird hier nicht konsequent Rechnung getragen. Ein weiterer Kritikpunkt liegt in der Betonung der unter-schiedlichen Aspekte «Gedanken - Gefühle - Verhalten». Zu Recht betonen die Autoren die oftmals mangelhafte Würdi-gung emotionaler Aspekte in der Verhaltenstherapie. Durch ihre Darstellung von Gedanken und Gefühlen als reinen «Folgeerscheinungen» in Kapitel 7 laufen sie jedoch nicht minder Gefahr, genau diesen Fehler zu wiederholen. Dies setzt sich in den nachfolgenden Kapiteln 
fort, in denen sie viel Wert auf die Gestaltung von Rahmenbedingungen sowie auf Verhaltensaspekte und Kognitionen legen. Emotionen behal-ten weiterhin eher den Status von «Begleitumständen». Sehr gut ist dagegen die Hinführung zur Zielanalyse in Kapitel 9, die der vorherigen Verhaltensanalyse folgt. Doch auch hier könnte der innere Zusammenhang der Kapitel - unter ande-rem auch der nachfolgenden Kapitel 10-13, die darauf direkt Bezug nehmen - klarer dargestellt werden. Diese Kapitel gehen nun dezidiert auf die Bestandteile der SORCK-Gleichung bzw. auf konkrete Techniken der Pro-blembewältigung ein. Eindrucksvoll ist hier die eingängige, praxisnahe Darstellung der Techniken an umfangreichen Bei-spielen, wie man sie sich in früheren Kapiteln gewünscht hätte. Insbesondere die schwierige Aufgabe, den Leser gegenüber seinen kognitiven Fehlern und irrationalen Schlußfolgerungen zu sensibilisieren, wird hervorragend gelöst. Sehr gut auch die Einführung in die Disputationsphase als dem zentralen Aspekt der folgenden kognitiven Therapieschritte. Damit und mit der Einführung in die Technik der «Selbstinstruktion» werden dem Leser zwei wesentliche Ansatzpunkte für die Verände-rung von problematischen Kognitionen an die Hand gegeben. In Relation zum ersten Teil dürfte man jedoch das «Selbst-instruktionstraining» etwas kürzen und konkretisieren. Demgegenüber fällt die Qualität der Darstellung in Kapitel 11 drastisch ab. Es fällt schwer, die angekündigte Darstellung der emotionalen Aspekte zu finden. Vielmehr beläuft sich Kapitel 11 auf eine Erklärung der Veränderungsmöglichkeiten durch Lernprinzipien der Klassischen Konditionierung bzw. auf eine Erklärung der konstruktiven Nutzung dieser Prin-zipien. Im ganzen Kapitel herrscht außerdem Begriffsver-wirrung, da hier Therapietechniken unter lerntheoretischen Oberbegriffen dargestellt werden, wie z.B. die Methode der Reizkonfrontation unter dem Prinzip der Löschung. Generell konzentriert sich dieses Kapitel allein auf «Angst» und sollte daher besser explizit auch in der Überschrift darauf Bezug nehmen. Andere Emotionen werden völlig vernachlässigt. Auch entsteht das Bild, Emotionen würden ausschließlich auf der Basis Klassischer Konditionierung ruhen! Sehr schön dagegen die Darstellung der Technik der Progres-siven Muskelrelaxation, wenn auch das Bindeglied zum vorherigen Fließtext fehlt. Querverweise wären hilfreich, z. B. auf die Darstellung der Makroanalyse. Dasselbe gilt für die Darstellung der Systematischen Desensibilisierung, die ebenfalls isoliert neben den anderen Abschnitten des Kapitels steht. Sehr viel besser gelöst wird das Problem der praktischen Urn-setzung (Kap. 12). Die Leser werden mit praktischen Tips unterstützt und sehr praxisnah in die Technik des Rollenspiels mit all seinen Möglichkeiten eingeführt. Dieses Kapitel sollte

modellhaft für die übrigen stehen. Sehr gut ist die Darstellung der flexiblen Einsatzmöglichkeiten der beschriebenen Techniken an Beispielen der Angstbewältigung. Auch das nachfolgende Kapitel 13 erklärt in verständlicher Form die Technik des Kontingenzmanagements. Die Einsicht des Lesers in die vielfältigen Zusammenhänge seiner Proble-matik und vor allem in seine Attributionen wird entscheidend gefördert. Hier geben die Autoren nun auch die Literaturhin-weise, die bereits in früheren Kapiteln erforderlich schienen. In den Kapiteln 14 und 15 werden die Verhaltens- oder Pro-blemanalyse und die Therapiemethoden im dezidierten Be-handlungsplan logisch zusammengeführt. Der Leser erhält sehr gute und praktikable Hilfen zur genauen Spezifizierung des gewünschten Zielverhaltens. Sehr gut auch die praktischen Tips für die Gruppensitzungen, z. B. die vielfältigen Nutzungs-möglichkeiten von Modellen. Die Motivation des Teilnehmers wird gefördert durch die Vermittlung von Einsicht und des Gefühls der Eigenverantwortlichkeit. Schade, daß dies erst hier geschieht! Neben der Motivierung erscheinen auch die Techniken der Vorstellungsübung einerseits und der Vertrags-formulierung andererseits als sehr hilfreich, um die Erfolgs-chancen des Projekts zu maximieren. Dennoch bleibt durch die einfühlsame Darstellung der Autoren dem Leser der Rückweg offen (Kap. 16). Es wird ihm letztlich ermöglicht, die Umsetzung des erarbeiteten Plans abzubrechen ohne die Attribution des Versagens.

Alles in allem haben die Autoren ein brauchbares Manual vor-gelegt, um den Studenten ein Selbsterfahrungsprojekt zu er-möglichen, das zweifellos ihren praktischen Erfahrungsschatz erweitern wird. Davon ist sekundär eine Qualitätsverbesse-rung zukünftiger eigener Therapien zu erhoffen. Ein ähnliches Projekt ware insofern in der Tat auch für die Erstellung von Selbsterfahrungscurricula in der VT-Weiterbildung diplo-mierter Psychologen sinnvoll. Dafür bedürfte das vorgelegte Manual jedoch einer gründlichen Überarbeitung, zu der man die Autoren durchaus ermutigen sollte.

D. Laubenstein, Mannheim

Körkel, /., Lauer, G., Scheller, R. (Hrsg.)

Sucht und Rückfall. Brennpunkte deutscher Rückfall-

forschung

Stuttgart, Enke, 1995224 S., DM 68-ISBN 3-432-26551-4

In der somatischen Medizin müssen Arzt und Patient meist ak-zeptieren, daß «Heilung» häufig nur eine relative oder teilweise Wiederherstellung sein kann. Dagegen wird von Psychiatrie und Klinischer Psychologie oft ein mehr oder weniger vollständiger Erfolg erwartet. Eine Enttäuschung dieser Hoffnungen kann dann entsprechend der Rollenverteilung der Beteiligten als «Unfähigkeit» oder «Uneinsichtigkeit» ausgelegt werden. Rückfälle machen den (vermeintlichen) Mißerfolg einer Be-handlung offenkundig. Rückfallattributionen können mit im-pliziten Krankheitsmodellen korrespondieren oder auch mit der aktuellen Rechtsprechung: Ein alkoholabhängiger Arbeit-nehmer, der nach einer Entwöhnungsbehandlung aus «Uneinsichtigkeit» rückfällig wird, hat in der Regel keinen Anspruch

52

Verhaltenstherapie 1996;6:51-55

Neuigkeiten vom Buch- und Zeitschriftenmarkt

auf Lohnfortzahlung oder Weiterbeschäftigung. Für Sucht-patienten und -therapeuten sind Rückfälle eher Regel als Ausnahme, und die Frustrationstoleranz beider Seiten wird dadurch immer wieder beansprucht. Seit einigen Jahren ist die Rückfälligkeit bei Abhängigkeitserkrankungen nun auch Gegenstand verstärkter Forschungsanstrengungen. Der vorlie-gende Band bietet auf 206 Seiten einen aktuellen Querschnitt deutscher Rückfallforschung. Die insgesamt 12 Beiträge wid-men sich den Themen Diagnostik, Prävention, Häufigkeit und Verlauf von Rückfällen, stationäre Rückfälligkeit, Rückfall-bewältigung durch Suchttherapeuten sowie Rückfalltheorien, wobei das Interesse der meisten Autoren hauptsächlich der Alkoholabhängigkeit gilt. Ergänzt werden die empirischen Beiträge durch zwei Übersichtsarbeiten. Breiten Raum nimmt die Darstellung und Überprüfung der sozial-kognitiven Rück-falltheorie von Marlatt ein, die auch in Deutschland intensive Forschung angeregt hat. Marlatt [1985] nimmt an, daß ein un-ausgewogener Lebensstil, der durch ein fehlendes Gleich-gewicht von Alltagsanforderungen einerseits und Möglich-keiten zur Entspannung andererseits gekennzeichnet ist, die Wahrscheinlichkeit erhöht, in Risikosituationen, z.B. bei un-angenehmen Gefühlszuständen oder zwischenmenschlichen Konflikten, auf Alkohol zurückzugreifen, um Spannungen zu reduzieren. Darüber hinaus seien fehlende oder unzureichende Bewältigungsmöglichkeiten, geringe Selbstwirksamkeits- sowie positive 
Alkoholwirkungserwartungen ungünstig für die Prognose. Bei erneutem Substanzgebrauch entscheide das Aus-maß des sogenannten Abstinenz-VerletzungsEffekts darüber, ob auf einen einmaligen «Ausrutscher» («lapse») ein schwerer Rückfall («relapse») folge. Die Gefahr eines Rückfalls sei besonders groß, wenn eine ursprünglich stark ausgeprägte Selbstverpflichtung zur Abstinenz mit einem ungünstigen internalen, stabilen und globalen Attributionsmuster (z.B. «Willensschwäche») einhergehe; aufkommende Schuld- und Schamgefühle seien dann Mitursache für verstärktes Trinken. Marlatts Theorie genießt große Popularität und wird in der kli-nischen Praxis wahrscheinlich vielen Patienten als plausibles Krankheitsmodell angeboten. Allerdings konnten zentrale Konstrukte der Theorie bisher nicht oder nur mit großen Ein-schränkungen empirisch bestätigt werden. Insofern fügen sich die referierten Ergebnisse gut in die derzeitige Befundlage. Wie auch schon andere Autoren zuvor, finden Sohns und Lauer in ihren Daten keine Hinweise auf einen Abstinenz-VerletzungsEffekt, während Schindler und Körkel lediglich das von Marlatt vermutete charakteristische Attributionsmuster (internal, stabil, global), nicht aber den Einfluß emotionaler Reaktionen (Schuld- und Schamgefühle) belegen können. Neben originär im Kontext klinischer Forschung entwickelten Rückfallmodellen dienen aber auch Beiträge anderer psycho-logischer Disziplinen als theoretischer Rahmen: Theorie ge-planten Verhaltens (Jonas und Eder-Jonas), Forschungsprogramm Subjektive Theorien (Sohns und Lauer), Weiners Attributionstheorie (Körkel und Wagner). Methodische Pro-bleme, z. B. die Operationalisierung von Rückfällen, theoretisch sinnvolle Subgruppenbildungen (Funke et al.) etc. werden im ersten Kapitel (Rückfallhäufigkeiten und -verläufe) behandelt. Zwar förderte die Einführung der «Dokumentationsstandards für die Behandlung von Abhängigen» eine Vereinheitlichung der Anamnese- und Katamneseerhebung, doch erscheint eine

weitergehende Standardisierung der Erhebungs- und Auswer-tungsmethoden nach wie vor wünschenswert. So können z. B. Scheller et al. zeigen, daß die Rückfallquote in Abhängigkeit vom jeweiligen Berechnungsmodus erheblich variieren kann. Ergebnisse einer multizentrischen Studie, die Effekte verschiedener Behandlungsmerkmale, z.B. allgemeiner und sucht-mittelspezifischer Kontrollmaßnahmen oder des zeitlichen Aufwands für Gruppentherapie und Realitätstraining, an einer großen Anzahl von Patienten überprüft, werden von Küfner et al. vorgestellt. Die Autoren heben besonders hervor, daß die Bewertung der einzelnen Behandlungsfaktoren von der Wahl der Erfolgsmaße abhängt: Eine geringere Rückfallquote wird in der Regel von einer erhöhten Abbruchquote begleitet. Den Herausgebern ist es bei der Auswahl der Beiträge gelun-gen, ein breites Spektrum aktueller Forschungsaktivitäten abzudecken, dennoch können einige Themen nur in den ab-schließenden Übersichtsarbeiten (Körkel und Lauer; Min-neker-Hügel) diskutiert werden, so z.B. die für weitere Forschung besonders wichtige Frage nach den Gemeinsamkeiten und Unterschieden der verschiedenen Abhängigkeitserkrankungen. Kann z.B. die Nikotinsucht als Modell für andere Substanzabhängigkeiten dienen? Welche Mechanismen der Entstehung und Aufrechterhaltung sind alien oder verschiedenen Abhängigkeitserkrankungen gemeinsam? Welche Paral-lelen können zwischen dem Rückfallgeschehen bei Substanz-abhängigkeit und anderen Störungen, beispielsweise schizo-phrenen Erkrankungen, gezogen werden? Diese und andere mögliche Fragen führen unmittelbar zu der Notwendigkeit, die Forschungsergebnisse anderer Disziplinen einzubeziehen. Körkel und Lauer verweisen in ihrem Literaturüberblick zwar auf genetische und neurobiologische Ansätze, berücksichtigen dabei aber weder neuere Tiermodelle des Verlaufs von Abhängigkeitserkrankungen [z.B. Wolffgramm, 1991] noch psychophysiologische Befunde [Überblick z.B. bei Newlin und Thomson, 1990], die als Hinweis auf einen biologischen Marker des Rückfallrisikos interpretiert werden können. Auf die Be-deutung soziodemographischer Variablen wird lediglich im Rahmen einer kurzen Zusammenfassung eingegangen. Große Teile der Sucht- und Rückfallforschung sind heute einer persönlichkeitspsychologischen bzw. typologischen Forschungs-strategie verpflichtet (z.B. Funke et al.), eine weitergehende Integration epidemiologischer, biologischer und psychophysio-logischer Befunde sowie eine stärkere Anknüpfung an klinischpsychologische und psychiatrische Forschung [z. B. Morey und Blashfield, 1991] scheinen notwendig. Die Ergebnisse einer Verlaufsuntersuchung von Küfner et al. [1988] legen beispielsweise nahe, die prognostische Relevanz differentialpsycholo-gischer Variablen zurückhaltend zu bewerten: Weder Persönlichkeitsmerkmale noch Angaben aus der Suchtanamnese, sondern ausschließlich soziodemographische Variablen, z.B. Beschäftigung, Wohnsituation und Partnerschaft betreffend, korrelierten mit dem katamnestischen Status 4 Jahre nach Entlassung aus einer stationären Behandlung. Der vorliegende Band belegt, obwohl einige Arbeiten vorwie-gend explorativen oder hypothesengenerierenden Charakter haben, eindrucksvoll die Fortschritte der deutschen Rückfallforschung, beispielsweise die Hinwendung des Forschungs-interesses zu Behandlungsmerkmalen (Küfner et al.). Maß-geblich die Beiträge des ersten Kapitels (Rückfallhäufigkeiten

Neuigkeiten vom Buch- und Zeitschriftenmarkt

Vcrhaltenstherapie 1996;6:51-55

53

und -verläufe) dokumentieren, daß sich innerhalb der Rück-fallforschung ein kritisches Methodenbewußtsein etabliert, das den komplexen Fragestellungen angemessen ist. Theore-tikern und interessierten Praktikern wird ein repräsentativer Überblick der deutschsprachigen Rückfallforschung geboten, wobei angesichts der Datenfülle eine Einordnung der Befunde möglicherweise leichter ist, wenn beim Lesen die abschließende Literaturübersicht vorangestellt wird. Leser, die unmittelbar verwertbare Handlungsanleitungen für die klinische Praxis suchen, werden auf andere Literatur zurückgreifen müssen -wahrscheinlich würden ohnehin viele Suchttherapeuten bei ihren Patienten nur ungern eine Tendenz zur externalen Ur-sachenattribution fördern.

Literatur

Deutsche Gesellschaft für Suchtforschung und Suchttherapie: Dokumentationsstan-dards 2 für die Behandlung von Abhängigen. Freiburg i. Br., Lambertus, 1992. Küfner H, Feuerlein W, Huber M: Die stationäre Behandlung von Alkohol-abhängigen: Ergebnisse der 4-Jahres-Katamnesen, mögliche Konsequenzen für Indi-kationsstellung und Behandlung. Suchtgefahren 1988;34:157-271. Marlatt GA: Relapse prevention: Theoretical rationale and overview of the model; in Marlatt GA, Gordon JR (eds): Relapse Prevention: Maintenance Strategies in the Treatment of Addictive Behaviors. New York, Guilford, 1985. pp 3-70.

Morey LC, Blashfield RK: Empirical classifications of alcoholism. J Stud Alcohol 1981;42:925-937.

Newlin DB, Thomson JB: Alcohol challenge with sons of alcoholics: A critical review and analysis. Psychol Bull 1990;108:383-402.

Wolffgramm J: An ethopharmacology approach to the development of drug addiction. Neurosci Biobehav Rev 1991;15:515-519.

Raíj Demmel, Mannheim

Krauß, H.

Verhaltensmedizin und Verhaltensanalyse 
München, Quintessenz, 1993194 S., DM58,-ISBN 3-86128-126-0

Die Problem- und Verhaltensanalyse hat zum Ziel, psychische Probleme oder Störungen, derentwegen Menschen einen Psychotherapeuten aufsuchen, zu beschreiben, zu klassifi-zieren und jene Faktoren bzw. Mechanismen zu identifizieren, die die Entstehung und Aufrechterhaltung der Probleme er-klären können. Auf der Basis einer solchen Analyse erfolgt die Indikationsstellung für oder gegen eine psychologische Therapie. Ist die Indikation für eine psychologische Therapie gegeben, werden unter Berücksichtigung der Zielvorstellun-gen des Patienten die therapeutischen Interventionen abge-leitet und durchgeführt.

Harald Krauß entwirft ein verhaltensmedizinisches Wirkmodell (VMW-Modell), mit dessen Hilfe psychische Störungen auf der Basis der Wechselwirkungen von physiologischen und psychischen Prozessen sowie im Zusammenwirken mit Ver-haltensparametern beschrieben und erklärt werden sollen. Der Autor will mit seinem Modell die Einseitigkeiten früherer Verhaltensanalyse-Modelle überwinden und praktisch tätigen Therapeuten eine plausible Orientierungshilfe für Diagnostik, Indikation und Therapie bieten.

Im 1. Kapitel werden bisherige Modelle zur Verhaltensanalyse exemplarisch dargestellt und kritisch bewertet. So bedauert der Autor, daß die derzeit gängigen Modelle nicht in der Lage sind, Phänomene wie Verdrängung, Symptomwechsel, Wider-stand sowie den Zusammenhang von psychischen und physi-schen Reaktionen zu erklären.

Im 2. Kapitel stellt der Autor in einem Überblick die Kompo-nenten seines verhaltensmedizinischen Wirkmodells vor. Das Modell sieht die Unterscheidung zwischen strukturellen und situativen Komponenten vor. Auf der situativen Ebene wird unterschieden zwischen Stimulus, Stimulusbewertung, Aktivie-rung, Akîivierungstransfer und Verhaltensreaktion. Die struk-turelle Ebene sieht die Komponenten struktureller Stimulus, Selbst-Struktur, Aktivierungsmuster, Transfermuster und Ver-haltensmuster vor.

In den folgenden Kapiteln werden die einzelnen Komponenten erläutert, und es werden jeweils Schlußfolgerungen für die klinische Praxis abgeleitet. So werden im 3. Kapitel Stimuli als Ergebnisse innerhalb oder außerhalb der Person definiert, die je nach Art der Bewertungen zu unterschiedlichen Aktivierungen im vegetativen Nervensystem führen und mit Verhal-tensreaktionen verbunden sind, die wiederum zum Stimulus werden (können). Als strukturelle Stimuli werden Stimuli der Vergangenheit oder Gegenwart bezeichnet, die über längere Zeit auf das Individuum einwirken oder eingewirkt haben (z.B. Sozialisations- und Konflikt-Umwelten). Im 4. Kapitel geht der Autor auf die Komponente Stimulus-Bewertung ein. Er unterscheidet zwischen einem Bewertungs-prozeß und einer Selbst-Struktur, die wiederum die Komponenten Stimulus-Norm (als «angeborene» Normen) und Stimulus-Inventar umfassen soil. Hinsichtlich der Stimulus-Normen wählt der Autor vier Normen aus (Orientierung; Kontrolle; Soziale Nähe; Gute Gestalt), die evolutionstheoretisch betrachtet für das Überleben der Mehrheit relevant sein sollen. Der Autor weist allerdings zu Recht darauf hin, daß die Auswahl gerade dieser Normen letztlich als vorläufig bzw. potentiell erweite-rungsbedürftig aufzufassen ist. Dennoch können die Überle-gungen des Autors unter heuristischer Perspektive für die klinisch-psychologische Praxis nützlich sein. So erläutert er anhand von Beispielen, inwiefern die Verletzung der genannten Normen zu bestimmten psychophysiologischen Aktivierungs-mustern und verhaltensmedizinischen Problemen führen kann. Problematisch erscheint allerdings, daß die Ausführungen des Autors den Eindruck erwecken, daß bereits durch die Definition (oder Annahme) bestimmter Normen bzw. Normenver-letzungen klinisch-psychologische Phänomene erklärt werden könnten. So weist er z. B. darauf hin, daß die Definition einer «Nahrungs-Norm» zur Erklärung von Eßstörungen beitragen könnte. Durch die Konstatierung von Normen oder Normen-verletzungen kann allerdings letztlich noch nicht davon ge-sprochen werden, daß die Mechanismen, die zur Entstehung bzw. zur Aufrechterhaltung eines psychischen oder verhaltensmedizinischen Problems beitragen, aufgeklärt worden wären. Unter dem Stimulus-Inventar will der Autor eine Ansamm-lung von konditionierten Stimuli verstanden wissen, die im Verlauf der individuellen Lerngeschichte in «Auseinanderset-zung» mit der Stimulus-Norm entstanden sein sollen. Was damit allerdings letztlich genau gemeint sein und welchen Erklärungswert dieses Konstrukt haben soil, bleibt unklar. So trägt die verwendete Terminologie (z. B. «Das Stimulus-Inventar (S-Inv) kann sich gegen die Person richten...» [S. 62]) meines Erachtens kaum zur Klärung des Konstruktes bei. Vielmehr deutet sich hier - wie auch bei der Art der Verwen-dung anderer Begrifflichkeiten - die Gefahr der Reifikation von Konstrukten an.

54

Verhaltenstherapie 1996;6:51-55

Neuigkeiten vom Buch- und Zeitschriftenmarkt

Das 5. Kapitel beschäftigt sich mit der vegetativen Aktivierung. Es wird angenommen, daß es zu einer Aktivierung des vegetativen Nervensystems kommt, sofern ein Stimulus als relevant für die Person bewertet wird. Die vegetative Aktivierung soil dem Konzept zufolge in Verhaltensreaktionen transferiert werden, was der Autor als Aktivierungstransfer bezeichnet. Zu psychischen Störungen soil es dem Modell zufolge dann kommen, wenn die Regulationsprozesse des vegetativen Nervensystems längere Zeit beeinträchtigt werden. Im 6. Kapitel beschreibt der Autor seine Vorstellungen darüber, wie die vegetativen Aktivierungen in Verhaltensmuster übertra-gen werden. Vor dem Hintergrund des Aktivierungskonzepts schlußfolgert er, daß erst Behandlungen über einen mehrmona-tigen Zeitraum Veränderungen der Aktivierungsmuster und des Verhaltens erlauben. Die Argumentation wird allerdings lediglich durch eine schmale Datenbasis abgesichert. In den Kapiteln 7 und 8 werden verschiedene Verhaltensreaktionen (z. B. situative Ebene) und Verhaltensmuster (z. B. struk-turelle Ebene) beschrieben und therapeutische Implikationen abgeleitet. Im Abschnitt über inneres Verbalverhalten fällt posi-tiv auf, daß der Autor die Wichtigkeit von privaten Theorien der Patienten im Hinblick auf die Verhaltensanalyse und The-rapie hervorhebt. Dem Autor ist grundsätzlich zuzustimmen, daß den subjektiven Annahmen und Überzeugungen der Patienten in älteren therapeutischen Ansätzen viel zuwenig Beachtung geschenkt wurde. Inwiefern die Berücksichtigung -und gegebenenfalls Veränderung - subjektiver Überzeugungs-systeme der Patienten allerdings notgedrungen «viel» Zeit er-fordert, wie der Autor verschiedentlich betont, ist letztlich eine empirische Frage.

In den beiden letzten Kapiteln geht der Autor auf die Bedeu-tung von Selbstwirksamkeit und Orientierung für den Patienten und für den Therapeuten ein In der Gesamtbewertung des verhaltensmedizinischen Wirk-modells ist dem Autor grundsätzlich dahingehend zuzustimmen, daß verhaltensmedizinische Befunde viel starker als bisher bei der Problem- und Verhaltensanalyse Berücksichtigung finden sollten. Positiv hervorzuheben ist auch, daß der Autor seine Sichtweise immer wieder durch konkrete Fallbeispiele illu-striert und durch empirische Befunde zu stützen versucht. Kri-tisch anzumerken ist allerdings, daß 
die verwendeten Begriff-lichkeiten nicht immer hinreichend präzise definiert werden und daß die für die Argumentation herangezogene Datenbasis teilweise recht schmal ist und/oder nicht immer dem aktuellen Forschungsstand entspricht.

In der aktuellen Diskussion zu Problem- und Verhaltensanalyse-Konzepten wird ferner die Frage diskutiert, ob allgemeine,störungsunspezifische Analysemodelle dem heutigen wissen-schaftlichen Standard noch angemessen sind. Es wird zuneh-mend dafür plädiert, störungsspezifische Modelle anstelle vonallgemeinen Analysemodellen anzuwenden, sofern es sich beiden Problemen eines Patienten um eine bereits gut erforschteStörung handelt. Wünschenswert ware es daher meines Erach-tens gewesen, wenn der Autor sein verhaltensmedizmischesModell in stärkerem Maße auf spezifische verhaltensmedizinische Probleme zugeschnitten und vor dem Hintergrund desaktuellen Forschungsstands jene therapeutischen Interventio-nen beschrieben hätte, die sich bei diesen Problemen empirischbewährt haben.

B. Tuschen, Marburg

\section{Kognition}

Imagination

Hypnose

Fachtagung vom 28.4. bis 1.5. 96

Vorträge

Workshops

Demonstrationen

Unterschiede und Geineinsamkeiten der verschiedenen Verfahren

Anwendungen bei imterschiedlichen Störimgsbildern

Erfahrene Therapeuten des In- und Auslands

, STUTTGARTER

THERAPIETAGE ' 96

Christophstraße 8, 70178 Stuttgart, Tel. 0711/640 8092

Fax 0711/640 8093

1Ojah

$1 / 8$ Ẅtagu $\notin$ reIFKV

Die Rolle der Person in der Verhaltenstherapie

12. Oktober 1996 Bad Dürkheim

Festakt

Wissenschaftliche Tagung:

Philosophische Definition des Begriffes Person

Einmaligkeit und WiUensfreiheit in der Psychologic

Person und Angsttherapie

Person und Persönlichkeitsstörungen

Personenorientierte Verhaltenstherapie

1 Abend: $\uparrow \varphi \varphi S_{i}$.

Ausführliches Programm und Anmeldung:

Institut für Fort- und Weiterbildung in klinischer Verhaltenstherapie e. V.

Kurbrunnenstr. 21a, 67098 Bad Dürkheim

Tel: (0 63 22) 680 19, FAX: (0 63 22) 68010 